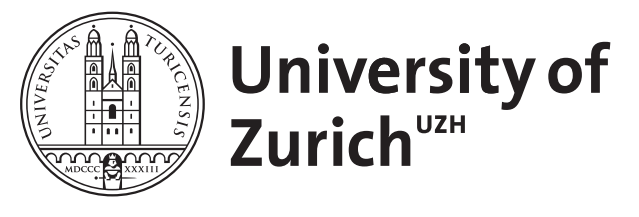

\title{
Lebensstile und Wohnstandortwahl
}

Rössel, Jörg ; Hölscher, M

\begin{abstract}
In der Lebensstilforschung und verschiedenen Disziplinen der Raumforschung wird die These vertreten, dass Lebensstile relevant für Entscheidungen in unterschiedlichen Verhaltensbereichen, insbesondere aber im Feld der Auswahl von Wohnungen und Wohnstandorten sind. Die empirische Befundlage zu dieser Behauptung ist allerdings ausgesprochen uneinheitlich. Um die Bedingungen für die Relevanz von lebensstilbasierten Präferenzen für die Wohnungs- und Wohnstandortwahl zu präzisieren, knüpft dieser Aufsatz an ein einfaches entscheidungstheoretisches Modell an, das sowohl in der Stadtsoziologie wie auch in der Lebensstilforschung Verwendung gefunden hat. Darauf aufbauend wird eine empirische Studie auf der Basis einer Quartiersbefragung in Leipzig präsentiert, die den Einfluss von Lebensstilen auf die Wohnungs- und Wohnstandortwahl unter Bedingungen und in einem Kontext prüft, die eine besonders große Relevanz von Lebensstilen erwarten lassen. Insgesamt zeigen aber die Ergebnisse, dass lebensstilbasierte Präferenzen selbst unter diesen Bedingungen nur eine beschränkte Erklärungskraft aufweisen, während die Ressourcenausstattung von Personen und ihre Lebensform für Wohnentscheidungen von deutlich größerer Relevanz sind.
\end{abstract}

DOI: https://doi.org/10.1007/s11577-012-0166-5

Posted at the Zurich Open Repository and Archive, University of Zurich

ZORA URL: https://doi.org/10.5167/uzh-62992

Journal Article

Accepted Version

Originally published at:

Rössel, Jörg; Hölscher, M (2012). Lebensstile und Wohnstandortwahl. Kölner Zeitschrift für Soziologie und Sozialpsychologie, 64(2):303-327.

DOI: https://doi.org/10.1007/s11577-012-0166-5 


\section{Lebensstile und Wohnstandortwahl}

Jörg Rössel und Michael Hoelscher

\section{Einleitung}

In den verschiedenen Bereichen der Raumforschung, sei es der Stadtsoziologie, der Geographie oder der Verkehrsforschung, wird über eine Erweiterung des Erklärungsinstrumentariums diskutiert, wobei insbesondere dem Konzept der Lebensstile eine herausgehobene Position eingeräumt wird (Klee 2003; Schneider und Spellerberg 1999; Blasius und Friedrichs 2011; Brun und Fangani 1994). Den Hintergrund dieser Überlegungen bilden zumeist Annahmen über die Veränderung der Sozialstruktur von Industriegesellschaften in den vergangenen Jahrzehnten, die aus einer Zunahme des Wohlstands, einem Abbau von tradierten Verhaltensvorschriften und damit gewachsenen individuellen Freiheitsspielräumen resultieren (Klee 2003; vgl. Beck 1986; Schulze 1992). Vorgeschlagen wird daher eine Ergänzung der konventionellen Determinanten des räumlichen Verhaltens, seien es Klassen- und Schichtkonzepte oder soziodemographische Variablen durch Begriffe, die in stärkerem Maße die individuelle Gestaltbarkeit von Lebenssituationen in den Vordergrund rücken (Klee 2003; Ohnmacht et al. 2009; Scheiner 2006). Dies deckt sich mit dem klassischen Anspruch der soziologischen Lebensstilforschung, die auch die subjektive Gestaltbarkeit von Lebensstilen und deren Erklärungskraft über konventionelle sozialstrukturelle Kategorien hinaus betont (Otte 2005). Betrachtet man allerdings die bisherige empirische Forschung zu diesem Thema, so sind die Befunde ausgesprochen uneinheitlich. In zahlreichen Studien wird zwar die Relevanz von weichen (Lebensstil)Faktoren für die Standortwahl betont, aber nicht systematisch nachgewiesen (Brun und Fangani 1994; Hill und Wiest 2004; Wiest und Hill 2004a, 2004b; Wiest und Zischner 2006). Dagegen schätzen andere Autoren die Relevanz von Lebensstilen als eher geringfügig ein (Spiegel 2000; Heijs et al. 2009). Die vorliegenden empirischen Studien unterscheiden sich zudem relativ stark in ihren abhängigen Variablen und kommen daher zu uneinheitlichen Resultaten. Das überwiegende Resultat dieser Publikationen ist allerdings, dass Lebensstile nur eine und zumeist eine nachrangige Determinante der Wohnstandort- und Wohnungswahl darstellen (Ærø 2006; Otte 2004; Scheiner 2006; Spellerberg 2007).

Vor dem Hintergrund dieser uneinheitlichen Befundlage soll in diesem Aufsatz die Relevanz von Lebensstilen für das räumliche Verhalten von Personen neu konzipiert werden. Erstens indem das Konzept der Lebensstile in ein allgemeineres Modell des Entscheidungsverhalten eingebettet wird, das in ähnlicher Form sowohl in der Stadtforschung wie auch in der 
Lebensstilforschung verwendet wird (Friedrichs 1988, 2008; Rössel 2006a, 2008). Zweitens soll darüber hinaus präzisiert werden, welche Dimensionen von Lebensstilen überhaupt für die Erklärung der Wohnstandort- und Wohnungswahl relevant sind. Drittens wird die Relevanz von Lebensstilen in dieser Untersuchung am Beispiel von Daten aus einer Leipziger Studie geprüft werden, also unter Bedingungen eines ausgesprochenen Mietermarktes, so dass Lebensstileffekte besonders stark hervortreten sollten. Die theoretischen Überlegungen werden in Abschnitt 2 vorgestellt und zur Entwicklung von empirischen Fragestellungen verwendet. Diese werden auf der Grundlage von Daten aus einer Quartiersstudie in Leipzig empirisch getestet. Die Daten und die Kontextbedingungen werden in Abschnitt 3 vorgestellt, in Abschnitt 4 werden die empirischen Resultate präsentiert. Insgesamt zeigt die Studie, dass selbst unter Bedingungen, in denen Lebensstile einen großen Einfluss haben könnten, die Bedeutung von Lebensstilen für die Auswahl von Wohnungen und Wohnstandorten eher von untergeordneter Bedeutung ist.

\section{Lebensstilforschung und Stadtsoziologie}

Die soziologische Lebensstilforschung ist in den achtziger Jahren mit dem Anspruch aufgetreten, traditionelle Konzepte der Analyse und Beschreibung von Sozialstrukturen zu ergänzen oder sogar zu ersetzen (Otte 2004, 2005). Den Hintergrund für diese Haltung bildete eine Unzufriedenheit mit ressourcenbasierten Ungleichheitskonzepten, wie sozialer Klasse und sozialer Schicht, die eine zufriedenstellende Erklärung von individuellem Verhalten kaum noch möglich erschienen ließen. Diese kritische Haltung gegenüber ressourcenorientierten und vertikal orientierten Konzepten entstand in den achtziger Jahren im Kontext von zwei zentralen Diskussionslinien in der Sozialstrukturanalyse: erstens der Individualisierungsthese, die aufgrund der Verbreitung von Massenwohlstand, gestiegener Mobilität und gewachsenen Bildungschancen Klassen- und Schichtkonzepten ihre Relevanz für die Analyse gegenwärtiger Gesellschaften abgesprochen hat (Beck 1986), zweitens Thesen der Differenzierung und Pluralisierung der Sozialstruktur, die den Bedeutungsgewinn neuer Ungleichheiten und die damit verbundene Inkonsistenz von Ungleichheitspositionen betont haben (Hradil 1987; Berger 1986). Die Lebensstilforschung reagierte auf diese Diskussionslage mit einem Konzept, das stärker die subjektive Verwendung von Ressourcen in das Zentrum der Analyse stellte und nicht mehr die Ressourcenausstattung selbst. Lebensstile sollten also insbesondere erklären können, warum Personen mit einer vergleichbaren sozioökonomischen Position deutliche Unterschiede in ihren Verhaltensmustern aufweisen. Daher sollten Lebensstile ihrem Anspruch nach über klassische 
sozialstrukturelle Variablen hinausgehend einen zusätzlichen Erklärungsbeitrag für unterschiedliche Verhaltensbereiche leisten.

Vor dem Hintergrund der Diskussion über unterschiedliche Definitionen von Lebensstilen in der Literatur wird hier folgende Bestimmung des Begriffs vorgeschlagen (vgl. Hartmann 1999; Otte 2005; Rössel 2005): Bei Lebensstilen handelt es sich um Muster von verschiedenen Verhaltensweisen, die eine formale, häufig ästhetische, Verwandtschaft aufweisen, daher zugrundeliegende Orientierungen zum Ausdruck bringen und von anderen Personen identifizierbar sind. Das tatsächliche Ausmass der inhaltlichen Kohärenz von Lebensstilen und ihre tatsächliche zeitliche Stabilität müssen aber als empirische Fragen betrachtet werden.

Lebensstile werden als Erklärungskonzept ergänzend $\mathrm{zu}$ traditionellen Begriffen der Sozialstrukturanalyse mittlerweile in zahlreichen sozialwissenschaftlichen Bereichen, von der Gesundheitsforschung (Klein et al. 2001; Abel et al. 2006), über die politische Soziologie (Mochmann und El-Menouar 2005; Otte 1997) bis hin zur Familienforschung (ArranzBecker und Lois 2010; de Haan und Uunk 2001; Klocke und Lück 2001) verwendet. Relativ frühzeitig wurde das Lebensstilkonzept auch in der sozialwissenschaftlichen Stadt- und Raumforschung berücksichtigt. So hat Blasius den Zusammenhang von Gentrifizierung und Lebensstilen schon zu Beginn der neunziger Jahre untersucht und Spellerberg hat gemeinsam mit Schneider dann Mitte derselben Dekade eine umfassende empirische Bestandsaufnahme des Zusammenhangs von Lebensstilen und Wohnen vorgenommen (Blasius 1993; Spellerberg 1997; Schneider und Spellerberg 1999). Mittlerweile liegen sowohl aus der Soziologie, als auch aus der Geographie und der Raumforschung zahlreiche Beiträge vor, die den Erklärungsbeitrag von Lebensstilen berücksichtigen (Beauregard 1986; Zukin 1989, 1998; Brun und Fangani 1994; Dangschat 1994; Bridge 2001; Klee 2003; Ley 2003; Ærø 2006; Kauko 2006; Scheiner 2006; Ohnmacht et al. 2009; Heijs et al. 2009; im Überblick Blasius und Friedrichs 2011). Dabei wird neben Analysen der Wohnungsausstattung und der räumlichen Mobilität insbesondere Fragen der Auswahl von Wohnungen mit bestimmten Merkmalen und an bestimmten Standorten eine besondere Bedeutung beigemessen. Diese beiden Explananda, Wohnstandortwahl und Wohnungswahl, sollen auch im Mittelpunkt des vorliegenden Beitrages stehen. So weisen Blasius (1993) sowie Büttner und Mühmer (2004) darauf hin, dass junge, hochqualifizierte Bewohner von innenstadtnahen Wohnquartieren häufig eine Präferenz für grosszügig geschnittene, ästhetisch anspruchsvolle Wohnungen mit flexiblen Grundrissen, abgeschliffenen Holzfussböden und alten Stuckdecken haben. Diese ästhetisierende und lebensstilbasierte Perspektive von Mietern und Käufern auf die 
Eigenschaften von Wohnungen wird auch von Bridge auf der Basis von Interviews mit Maklern bestätigt (2001). Geradezu prototypisch kristallisieren sich die genannten Merkmale in den sogenannten Loft Wohnungen heraus, also Wohnungen in ehemaligen Fabrikgebäuden, die für die Wohnnutzung umgewandelt wurden (Zukin 1989; Büttner und Mühmer 2004). An diesen wird auch die Bandbreite möglicher, lebensstilbasierter Nutzungsformen deutlich, da Lofts ein grosses Spektrum von Wohnformen ermöglichen, die von einer eher improvisierten Nutzung ehemaliger Fabrik- oder Lagerhallen durch Künstler und kulturelle Pioniere bis hin zu aufwändig und luxuriös sanierten Wohngebäuden mit höchstem Komfort reichen können (Büttner und Mühmer 2004; Podmore 1998).

Neben der lebensstilgesteuerten Auswahl von Wohnungen mit spezifischen Merkmalen wird in der Literatur auch die Wahl von Wohnstandorten auf der Basis von Lebensstilen diskutiert. Hier liegt der Fokus insbesondere auf Personengruppen mit ausgesprochen urbanen Lebensstilen, die verstärkt in die Innenstädte ziehen und damit Prozesse der Reurbanisierung und Gentrifizierung antreiben. Unter Gentrifizierung wird eine soziale und bauliche Aufwertung von Stadtteilen verstanden (für Definitionen: Zukin 1989; Dangschat und Blasius 1990; Friedrichs 1998). Diese findet typischerweise in innerstädtisch gelegenen Altbauquartieren mit einem gründerzeitlichen Baubestand und niedrigen Mieten statt, die vor Beginn des Prozesses häufig eine sozial schwache Bevölkerung aus Arbeitern, Ausländern und Älteren aufweisen. Im Prozess der Gentrifizierung drängen sukzessive Gruppen von neuen Bewohnern in das Viertel ein. Handelt es sich im ersten Schritt um Personengruppen wie Künstler und Studenten auf der Suche nach günstigem und attraktivem Wohnraum, so folgen im zweiten Schritt besserverdienende Bevölkerungsgruppen mit urbanen Lebensstilen und Haushaltsformen, also häufig unverheiratet zusammenlebende Paare ohne Kinder, die einen innerstädtischen Lebensstil pflegen wollen. Für diese zweite Gruppe ist das Viertel unter anderem auch aufgrund der Aktivitäten der ersten Gruppe attraktiv, die zur Ansiedlung interessanter Geschäfte, Galerien und Gaststätten auf der einen Seite und zur Renovierung zahlreicher Gebäude geführt haben. Die erstgenannte Gruppe wird in der Forschung häufig als Pioniere bezeichnet, während die zweite Gruppe zumeist als Gentrifier benannt wird.

Betrachtet man die skizzierten Diskussionen über die Auswahl von Wohnungen und Wohnstandorten, so fallen vor allem zwei lebensstilbasierte Mechanismen ins Auge, die immer wieder als zentral für diese Prozesse genannt werden. Während der erste vor allem auf eine Orientierung auf urbane Kultur verweist, fokussiert der zweite insbesondere auf die Hochkulturorientierung von Personen. Andere Lebensstildimensionen werden in der 
internationalen Literatur kaum diskutiert, so dass diese an dieser Stelle nicht weiter verfolgt werden. ${ }^{1}$

Erstens wird in zahlreichen Texten darauf hingewiesen, dass Personen räumlich situierte Angebote nutzen, um ihren jeweiligen Lebensstil ausleben zu können (Klee 2003: 70; Ærø 2006: 110; Beauregard 1986: 43 - 44; Scheiner 2006: 56 - 57; Friedrichs 2008: 395 - 396; Brun und Fangani 1993: 929 - 930; Kauko 2006). So wie ein heimzentrierter Lebensstil das eigene Haus mit Garten und Werkstatt benötigt, kann ein urbaner Lebensstil, der auf die Nutzung städtischer kultureller Einrichtungen und gastronomischer Betriebe ausgerichtet ist, nur in innerstädtischen Quartieren gelebt werden. Dies gilt sowohl für die oben genannte Gruppe der Gentrifier, als auch für die Gruppe der Pioniere, die beide die spezifisch urbanen Qualitäten von innerstädtischen Quartieren für ihre Lebensstile nutzen (Friedrichs 1998: 64). Zukin (1998) verweist darauf, dass mit dieser Perspektive auch eine veränderte Orientierung auf die Funktion von Städten deutlich wird, die immer stärker von Standorten der Produktion zu Standorten der Konsumtion werden. Häufig taucht im Zusammenhang mit diesem Mechanismus auch die These auf, dass bestimmte Lebensstilgruppen mit stark urbanen Lebensstilen die städtische Szenerie und ihre Wohnungen als Bühne für das Zelebrieren ihres Lebensstils verwenden (Dangschat 1994: 427; Beauregard 1986: 43 - 44; Klee 2003: 72). Dieser Mechanismus soll hier als urbane Kulturorientierung bezeichnet werden.

Zweitens wird aber neben der Nutzung des räumlich situierten Kulturangebots als Gelegenheitsstruktur zum Ausleben des eigenen, urbanen Lebensstils auch auf eine verallgemeinerte ästhetische Disposition verwiesen (Ley 2003: 2529). Die Diskussion knüpft hier insbesondere an Bourdieus Vorstellung an, dass die oberen Klassen sich durch eine spezifische ästhetische Einstellung auszeichnen, die es ihnen erlaubt nicht nur die Werke der legitimen Kultur (Hochkultur) unter ästhetischen Gesichtspunkten zu betrachten, sondern auch Produkte der Populärkultur, ja sogar Alltagsgegenstände zu ästhetisieren (Bourdieu 1982: 80). Insofern sollten Personen mit einem hochkulturellen Lebensstil ihre Wohnung und

\footnotetext{
${ }^{1}$ Dies hängt damit zusammen, dass die angelsächsische Lebensstilforschung sehr viel weniger als die deutschsprachige Forschung auf die Erfassung von Lebensstilen als holistischen Ganzheiten ausgerichtet ist und daher populärer Kultur in ihren verschiedenen Varianten eine geringere Aufmerksamkeit als der Hochkultur widmet, da diese für Fragen von sozialer Ungleichheit und kultureller Hegemonie als von größerer Relevanz erscheint (Otte und Rössel 2011: 10). Die Verwendung des Konzepts der „,kulturellen Allesfresser“ hat allerdings zu einer stärkeren Berücksichtigung von populärkulturellen Aspekten auch in der internationalen Lebensstilforschung geführt (vgl. Peterson und Kern 1996; Rössel 2006b). Dieser Begriff verweist auf die Idee, dass Personen mit einer Hochkulturorientierung in Gegenwartsgesellschaften typischerweise auch eine Vorliebe für eine größere Anzahl von nicht hochkulturellen Genres haben, also kulturell vielfältig orientiert sind, während andere Personen eher auf ein oder wenige Genres festgelegt sind (,univores“). Die in Abschnitt 4 berichteten empirischen Analysen wurden auch unter Berücksichtigung einer Variablen durchgeführt, die das Konzept der „kulturellen Allesfresser“ für die Befragten operationalisiert. Der statistische Einfluss dieser Variablen war noch weniger ausgeprägt als die Relevanz der Hochkulturvariablen, die in Abschnitt 4 berichtet werden.
} 
ihren Wohnstandort nicht allein aufgrund des jeweiligen kulturellen Angebotes auswählen, sondern auch auf Grundlage der ästhetischen Merkmale von Wohnung und Wohnumfeld, also beispielsweise der Architektur, der planerischen Gestaltung und der Ausstattung der Wohnung bzw. des Wohngebäudes, also z. B. der Wohnungsgröße, Holzfußböden und Stuckdecken (Ærø 2006: 119; Wiest und Hill 2004: 65; Thomas et al. 2008: 358; Bridge 2001). Dieser Mechanismus soll hier als hochkulturelle Ästhetisierung bezeichnet werden. Man kann an dieser Stelle fragen, ob dieser Mechanismus für die Gruppen der Pioniere und der Gentrifier in gleichem Maße plausibel ist, da diese sich in ihren soziodemographischen Merkmalen auf den ersten Blick deutlich unterscheiden. Während es sich bei den Pionieren um jüngere Personen mit hoher Bildung und niedrigem Einkommen handelt, werden die Gentrifier eher als Personen mittleren Alters, hohen Einkommens und einer mindestens mittleren Bildung betrachtet (Thomas et al. 2008; Friedrichs 1998). Die empirische Forschung hat gezeigt, dass die beiden wichtigsten soziodemographischen Determinanten einer Hochkulturorientierung erstens die Bildung und zweitens das Alter sind (Rössel 2005: 310 323; Otte 2005: 7; Schulze 1992). Sowohl mit der Höhe der Bildung als auch mit dem Alter, allerdings schwächer ausgeprägt, nimmt die Vorliebe für Hochkultur zu. Insofern ist für beide Gruppen, sowohl für die Pioniere, als auch für die Gentrifier eine Hochkulturorientierung zu erwarten.

Auf der Grundlage der skizzierten Thesen und Argumente erscheint die Relevanz von Lebensstilen für die Auswahl einer Wohnung mit bestimmten Eigenschaften und eines bestimmten Wohnstandortes als höchst plausibel. Auf der Grundlage der Literatur können aber eine Reihe von Argumenten gegen die beiden angesprochenen Mechanismen der Auswahl von Wohnungen und Wohnstandorten ins Feld geführt werden. So weisen Harth, Herlyn und Scheller (1998: 189) darauf hin, dass innenstadtnahe Kultur- und Freizeitangebote häufig von Personen genutzt werden, die überhaupt nicht in der Nähe dieser Einrichtungen wohnen. Auch Schulze (1994) vertritt die Behauptung, dass die Etablierung von kulturellen Szenen nicht an räumliche Nähe gebunden ist, so dass die Wohnstandortwahl keinen Zusammenhang mit den bevorzugten Lebensstilen aufweisen sollte. Diesen Thesen zufolge könnte ein urbaner Lebensstil relativ unabhängig vom Wohnstandort gepflegt werden, da die moderne Verkehrsinfrastruktur ein Erreichen von städtischen Freizeit- und Kulturangeboten zeit- und kostengünstig ermöglicht. Darüber hinaus finden sich in der Diskussion auch Argumente gegen den zweiten hier angesprochenen Mechanismus: so weist Kauko (2006) darauf hin, dass für die allermeisten Mieter und Käufer von Immobilien ästhetische Einstellungen eher wenig relevant sind, während ganz traditionelle Vorstellungen einer 
komfortablen und angemessen großen Wohnung für Auswahlentscheidungen wichtiger seien (vgl. auch Heijs et al. 2009).

Eine Reihe von empirischen Studien hat diese gegensätzlichen Thesen geprüft, wobei der Fokus zumeist auf der Wohnstandortwahl im engeren Sinne lag, weniger auf der Auswahl von Wohnungen mit spezifischen Merkmalen (vgl. aber Blasius 1993; Spellerberg 1997; Schneider und Spellerberg 1999). Die Befundlage ist durch uneinheitliche Ergebnisse geprägt. Spellerberg (2007) kann auf der Grundlage einer bundesweiten Befragung einen deutlichen Zusammenhang zwischen dem Lebensstiltypus einer Person und ihrem Wohnstandort in unterschiedlichen Gemeindetypen nachweisen. Dabei erweist sich der Lebensstil als wichtiger als traditionelle ressourcenbasierte Maße der sozioökonomischen Position wie soziale Schicht oder Einkommen, während der stärkste Einfluss auf den Wohnstandort von der Lebens- und Haushaltsform ausgeht. Auch Scheiner (2006) kann auf der Basis einer im Rheinland durchgeführten Studie zeigen, dass Personen mit einem Lebensstil der außerhäuslichen Selbstverwirklichung, der mit dem hier betrachteten Mechanismus der urbanen Kulturorientierung korrespondiert, häufiger in dicht besiedelten, innerstädtischen Quartieren mit einem vielfältigen Freizeit- und Einkaufsangebot leben. Dagegen äußern sich eine Reihe von anderen Autoren skeptisch über die empirischen Effekte von Lebensstilen auf die Wohnstandortwahl (Heijs et al. 2009; Spiegel 2000; Blasius und Friedrichs 2011). Hier ist insbesondere die Studie von Otte zu nennen, die die Relevanz von Lebensstilen für den Wohnstandort in Mannheim untersucht und zu dem Fazit kommt, dass diese über die traditionellen ressourcenbasierten Sozialstrukturkonzepte hinaus nur geringe Zusatzerkenntnisse liefern (Otte 2004: 283). Insgesamt ergeben also die vorliegenden Studien keine klaren Ergebnisse im Hinblick auf die Bedeutung von Lebensstilen für die Wohnstandortwahl. In den folgenden Abschnitten soll daher ein theoretisches Modell der Wohnstandortwahl skizziert werden, das auch Bedingungen für die Relevanz von Lebensstilen angeben kann.

Sowohl in der Stadtsoziologie (Friedrichs 1988; 2008, Blasius und Friedrichs 2011) als auch in der Lebensstilforschung (Rössel 2006a, 2008) sind entscheidungstheoretische Modelle vorgeschlagen worden, die den Einfluss von Lebensstilen auf Wohnstandortentscheidungen erklären können. Insbesondere Friedrichs (1988) hat dafür argumentiert, sozialräumliche Muster als kollektive Resultate individuellen Entscheidungsverhaltens zu betrachten und auf dieser Grundlage ein Modell der Wohnstandortwahl entwickelt (Friedrichs 1988; 2008; Blasius und Friedrichs 2011). Sein Modell der Wohnstandortwahl von Haushalten sieht einen zweistufigen, sequentiellen Entscheidungsprozess vor (Blasius und Friedrichs 2011: 410 - 
411). Der erste Filter in dieser Konzeption ist durch die Ressourcenausstattung der betrachteten Haushalte und die vorhandenen Handlungsalternativen definiert. So müssen sich Personen mit sehr niedrigen Einkommen in einem eher kleinen Segment des Wohnungsrespektive Immobilienmarktes ihre Wohnung suchen, während für Personen am oberen Ende der Einkommensverteilung nahezu alle auf dem Markt befindlichen Immobilien prinzipiell mögliche Optionen darstellen. Nur die im Rahmen der finanziellen Möglichkeiten stehenden Wohnstandorte werden im zweiten Entscheidungsschritt überhaupt berücksichtigt. Der zweite Filter beinhaltet die verschiedenen nutzenstiftenden Kriterien, nach denen die Haushalte die verfügbaren Wohnungen beurteilen. Dabei fokussiert Friedrichs vor allem auf die Ähnlichkeit der schon an einem Standort lebenden Personen mit den Merkmalen des wählenden Haushaltes. Dabei können Ähnlichkeiten der Familienstruktur, aber auch Lebensstilähnlichkeiten relevant sein. In diesem Modell ist zu erwarten, dass vor allem sehr gut mit Ressourcen ausgestattete Personen ihre Wohnungen mit einem gewissen Spielraum und daher lebensstilbasiert auswählen können, während dies für ressourcenschwache Haushalte nicht zutrifft. Diese müssen sich ausschließlich an den ökonomischen Notwendigkeiten orientieren.

In der Lebensstilforschung wurden vergleichbare entscheidungstheoretische Modelle vorgeschlagen (Rössel 2006a, 2008). Auch diese sehen vor, dass die Akteure ihre Präferenzen unter den durch ihre Ressourcenausstattung und die vorhandenen Handlungsalternativen gegebenen Restriktionen verfolgen. In zwei Hinsichten weicht dieses theoretische Modell, das in dieser Studie berücksichtigt wird, von dem skizzierten theoretischen Konzept von Friedrichs ab: erstens werden die Filter nicht zwangsläufig als sequentiell verstanden. Es wird davon ausgegangen, dass auch lebensstilbasierte Präferenzen von vorneherein die berücksichtigte Auswahl von Wohnungen einschränken können. Die subjektiv berücksichtigten Handlungsalternativen ergeben sich dann als Schnittmenge der beiden Filter. Zweitens wird nicht allein die Ähnlichkeit zwischen den wohnungssuchenden Haushalten und der potentiellen Nachbarschaft in das Zentrum gerückt. Die Präferenzen können daher unterschiedlicher Art sein: sie können im oben dargestellten Sinne lebensstilbasiert sein, sie können sich aber auch an der Lebensform orientieren, so dass Personen mit Kindern ein Haus mit Garten im Grünen vorziehen. In dieser Untersuchung werden die lebensstilbasierten Präferenzen der Akteure im Anschluss an die oben skizzierten zwei Mechanismen operationalisiert werden, d. h. es wird davon ausgegangen, dass die Akteure sich bei der Suche nach Wohnungen von der Stärke ihrer urbanen Kulturorientierung und ihrer Hochkulturorientierung leiten lassen. Beide Mechanismen stellen nicht die Ähnlichkeit 
zwischen den auswählenden Akteuren und den an einem Standort schon lebenden Personen in den Vordergrund, sondern die Korrespondenz zwischen den Lebensstilen und bestimmten Merkmalen der Wohnungen und der Wohnumgebung.

Es soll an dieser Stelle nicht unterstellt werden, dass die Akteure, den durch ihre Präferenzen definierten Nutzen im Rahmen ihrer Opportunitätsstruktur maximieren. Empirisch zeigt sich in der Regel, dass Personen selbst bei der Entscheidung über eine Wohnung nur wenige Alternativen in Betracht ziehen und dabei nur eine kleine Anzahl von präferenzbasierten Kriterien in Rechnung stellen (Friedrichs 1988; Friedrichs und Opp 2002). Es kann also zumindest unterstellt werden, dass Akteure ihre Präferenzen bei Wohnstandortentscheidungen berücksichtigen, ob sie dabei aber eine Maximierung vornehmen oder nur ein zufriedenstellendes Ergebnis (satisficing) erreichen wollen, soll an dieser Stelle nicht weiter betrachtet werden.

Auf der Grundlage dieses einfachen Modells kann nun präzisiert werden, unter welchen Bedingungen Lebensstile, bzw. lebensstilbasierte Präferenzen einen Einfluss auf die Wohnstandortwahl haben sollten und in welchen Kontexten sie wenig relevant sein sollten. Ein erster Punkt, der aus dem skizzierten entscheidungstheoretischen Modell folgt, ist, dass die Ausstattung und die Präferenzen von Personen für ihre Entscheidung nur dann einflussreich sind, wenn sich die Alternativen auch in relevanter Weise unterscheiden. So wird das Einkommen von Personen nur dann einen Einfluss auf ihre Wohnungsauswahl haben, wenn sich die Wohnungen in ihrer Miethöhe bzw. dem Kaufpreis unterscheiden (Friedrichs 1988: 66). Auch die hier im Mittelpunkt stehenden Lebensstile werden nur dann einen Einfluss auf die Wohnentscheidung haben, wenn sich die verfügbaren Wohnungen respektive die Wohnstandorte in lebensstilrelevanten Merkmalen unterscheiden. So können auch die unterschiedlichen Ergebnisse in den Studien von Spellerberg (2007) und Otte (2004) erklärt werden. Während Spellerberg unter anderem dörfliche und städtische Wohnstandorte vergleicht, die sich ganz offensichtlich in erheblichem Maße in ihren kulturellen und Freizeitangeboten unterscheiden, betrachtet Otte sechs Typen von Wohngebieten in der Stadt Mannheim, bei denen zumindest nicht offensichtlich ist, dass und wie sie in lebensstilrelevanter Weise differieren. Um also den Einfluss von Lebensstilen auf die Wohnungsauswahl zu prüfen, müssen Handlungsalternativen betrachtet werden, bei denen deutlich ist, dass sie sich in ihrer Lebensstilrelevanz unterscheiden. Dies gilt auch für den Einfluss anderer Determinanten, z. B. des Einkommens, das nur bei Handlungsalternativen, die tatsächlich in ihren Kosten differieren, relevant sein sollte. Aus dem entscheidungstheoretischen Modell folgt aber noch ein zweiter Punkt, der das Verhältnis der 
beiden angesprochenen Filter betrifft. Der erste angesprochene Filter beschränkt die Relevanz des zweiten Filters: nur Personen, die überhaupt über Wahlmöglichkeiten verfügen, können auf der Basis ihrer Präferenzen eine zufriedenstellende Wohnung aussuchen. Je grösser dieser ressourcendefinierte Handlungsspielraum ist, desto grösser sollte auch die Möglichkeit für eine präferenzgesteuerte Wahl einer Wohnung bzw. eines Wohnstandortes sein. ${ }^{2}$ An dieser Stelle wird deutlich, dass Lebensstile und ihre Berücksichtigung im Alltagsleben von Bedingungen sozialer Ungleichheit strukturiert sind. Da es sich bei Wohnungen um Güter mit relativ hohen Preisdifferenzen handelt, kann auf der Basis dieses entscheidungstheoretischen Modells generell ein eher geringer Einfluss von lebensstilbasierten Präferenzen auf die Wohnungs- und Wohnstandortwahl erwartet werden. Allerdings wurde diese Studie auf einem Wohnungsmarkt durchgeführt, der in hohem Maße den Kriterien eines Mietermarktes entspricht und daher stärker als andere Wohnungsmärkte Spielraum für die Effekte von Lebensstilen aufweisen sollte. Schließlich muss drittens auch noch darauf verwiesen werden, dass auch die Lebensstile von Personen so konzipiert sein müssen, dass eine präzise theoretische und interpretativ nachvollziehbare Verknüpfung zwischen Lebensstilen einerseits und Wohnungsmerkmalen andererseits hergestellt werden kann. Insbesondere bei typologisch angelegten Lebensstilkonzepten, die häufig auf der Basis einer Vielzahl von Indikatoren gebildet werden, ist dies kaum möglich, da hier nicht entschieden werden kann, welche der zugrundeliegenden Variablen tatsächlich für eine bestimmte Wohnstandortwahl relevant ist. Insofern liegt es nahe im oben skizzierten entscheidungstheoretischen Modell die Lebensstile als dimensionale Variablen abzubilden, die Präferenzen für bestimmte Eigenschaften von Wohnungen oder Wohnstandorten sinnverstehend nachvollziehbar beeinflussen (vgl. Rössel 2006a, 2008). Personen werden also nicht einem Lebensstiltyp zugeordnet, sondern es werden Lebensstilvariablen mit einem höheren Messniveau konstruiert und die Personen können für jede Variable eine tiefere oder höhere Ausprägung aufweisen (Otte 2005: 22 - 23). Bei der Konstruktion dieser Lebensstildimensionen orientiert sich die Studie an den beiden oben dargestellten Mechanismen der urbanen Kulturorientierung und der Hochkulturorientierung. In der folgenden empirischen Studie sollen die im vorhergehenden Absatz genannten drei Punkte ausdrücklich berücksichtigt werden, um die Relevanz von Lebensstilen für die Auswahl von Wohnungen respektive Wohnstandorten unter geeigneten Bedingungen zu prüfen. Dabei soll erstens die Frage betrachtet werden, ob Lebensstildimensionen, die den beiden oben skizzierten Mechanismen (Urbane Kulturorientierung, Hochkulturelle

\footnotetext{
${ }^{2}$ Dies spricht nicht zwangsläufig für eine Sequentialität der Filter. Auch bei geringen, ökonomisch definierten Wahlmöglichkeiten können von vorneherein bestimmte Wohnungen als nicht „lebensstiladäquat“ ausgeschlossen werden.
} 
Ästhetisierung) entsprechen, einen Einfluss auf die Wohnstandortwahl und die Auswahl von Wohnungen mit spezifischen Eigenschaften haben. Wie oben darstellt wurde, ist für die Gruppen der Pioniere und Gentrifier nicht allein der Fokus auf bestimmte Wohnstandorte, sondern auch auf Wohnungen mit spezifischen Merkmalen (Größe, Holzfussboden, Stuckdecken, Balkon) typisch, so dass neben den Standorten auch die Merkmale der Wohnungen berücksichtigt werden. Zweitens soll auch geprüft werden, ob die Möglichkeit für eine präferenzgesteuerte Auswahl einer Wohnung durch den ersten Filter, also insbesondere die Ressourcenausstattung einer Person, determiniert wird. Dies wird mit Daten über die von den Befragten selbst genannten Kriterien der Wohnungsauswahl erfasst. Insofern kann geprüft werden, ob Akteure mit einer besseren Ressourcenausstattung eine höhere Präferenzsteuerung in ihrer Wohnstandortwahl wahrnehmen.

\section{Daten und Methoden}

Die empirische Studie geht zurück auf ein Lehrforschungsprojekt, das im Jahr 2002 in einem Stadtteil im Leipziger Westen durchgeführt wurde und anschließend weitergeführt wurde. Es handelt sich hier also um eine Studie unter den spezifischen Bedingungen des ostdeutschen Wohnungsmarktes, der insbesondere für Personen mit einem mittleren bis hohen Einkommen ein ausgesprochenes Überangebot von Wohnungen ausweist (Steinführer 2004; Wiest und Hill 2004a; 2004b; Glatter und Killisch 2004; Wiest und Zischner 2006; Thomas et al. 2008: 359). Die Akteure sollten hier also in besonders hohem Maße präferenzgesteuert bei der Suche nach Wohnungen und Wohnstandorten vorgehen können (Hill und Wiest 2004a; 2004b). Daher stellt die vorliegende Studie keinen besonders konservativen Test des Einfluss von Lebensstilen dar, sondern findet in einem Kontext statt, der eine Präferenzsteuerung der Wohnstandortwahl und damit eine Relevanz von Lebensstilen gerade ermöglicht. Im Vergleich $\mathrm{zu}$ anderen räumlichen Kontexten sind hier also eher starke Effekte von lebensstilbasierten Präferenzen erwartbar. Damit kann die Untersuchung aber zentral zur Klärung der Rolle von Lebensstilen bei der Wohnstandortwahl beitragen: wenn selbst unter den relativ günstigen Bedingungen der hier untersuchten Quartiere keine oder nur schwache Lebensstileffekte auftreten, so ist dies in anderen Kontexten noch viel weniger zu erwarten. Im Rahmen des Projektes wurden in den Jahren 2002 und 2005 zwei schriftliche Befragungen von Personen in zwei ausgewählten Quartieren dieses Stadtteils durchgeführt. Die bisherige empirische Forschung zu Segregation und Aufwertung in Ostdeutschland hat gezeigt, dass sich derartige sozialräumliche Prozesse typischerweise sehr kleinräumig entwickeln (Wiest und Hill 2004; Buzar et al. 2007; Thomas et al. 2008: 360). Daher wurden zwei Quartiere 
ausgewählt, die sich jeweils um einen Straßenzug gruppieren und durch Verkehrsachsen und einen Fluss bzw. einen Kanal deutlich abgegrenzt sind. Es wurde ein Quartier berücksichtigt, das eine deutliche Aufwertungstendenz zeigt: zentraler architektonischer Anziehungspunkt ist eine gründerzeitliche Textilfabrik, die aufwändig für die Wohnnutzung umgewidmet wurde. Das Quartier ist geringfügig näher an der Innenstadt als das andere Quartier, so dass die Einrichtungen der urbanen Hochkultur, die sich im Stadtzentrum befinden, leichter erreichbar sind. Zudem liegt es somit auch deutlich näher am städtischen Grüngürtel, der die Quartiere des Leipziger Westens vom Stadtzentrum trennt. Die umgenutzte Textilfabrik und der zentrale Straßenzug des ausgewählten Quartiers liegen unmittelbar an einem Fluss. Im Quartier selbst gibt es gewisse Hinweise auf die für Aufwertungsprozesse typische Infrastruktur, vor allem im Bereich der Gastronomie. Neben mehreren Kneipen, die ein altersgemischtes, aber überwiegend jüngeres Publikum ansprechen, gibt es im Quartier auch mehrere Restaurants, die zum Teil auch gehobene Ansprüche bedienen. Eine Studie der gastronomischen Einrichtungen in den beiden Quartieren konnte zeigen, dass in den Gaststätten im Quartier mit Aufwertungstendenzen die Preise deutlich höher waren, das Angebot vielfältiger und anspruchsvoller und dass ein deutlich höherer Prozentsatz der Gäste Wein konsumierte (Rössel und Hölscher 2004). Darüber hinaus wurde über die Kneipen und Restaurants in diesem Quartier häufiger im Leipziger Stadtmagazin „Kreuzer“ berichtet, so dass der Aufwertungsprozess auch nach außen sichtbar wurde. Hinweise auf Aufwertungstendenzen im Bereich des Einzelhandels gab es allerdings kaum. Das andere berücksichtigte Quartier wird durch einen Kanal begrenzt, der durchaus hohe Freizeitqualitäten aufweist. Allerdings waren weder im Bereich der Gastronomie noch des Einzelhandels Aufwertungstendenzen sichtbar. Die gastronomischen Betriebe wurden durch Kneipen und Imbisslokale dominiert. Darüber hinaus waren auch architektonisch kaum Aufwertungstendenzen sichtbar. Es gab weniger Neubauten als im anderen Quartier und der Anteil nicht sanierter und unbewohnter Altbauten lag deutlich höher. Diese Unterschiede spiegeln sich auch in der Bevölkerungszusammensetzung der beiden Quartiere: während in dem Quartier mit Aufwertungstendenz lediglich $6 \%$ der Befragten arbeitslos waren, sind dies in dem anderen Quartier 20,4\%. ${ }^{3}$ Zusammengefasst zeigt diese kurze Skizze, dass die beiden ausgewählten Quartiere im Hinblick auf urbane Lebensstile ein deutlich differenziertes Angebot aufweisen, wobei das Quartier mit Aufwertungstendenzen eine architektonisch

\footnotetext{
${ }^{3}$ Diese Angaben entstammen der Befragung und nicht der amtlichen Statistik. Da beide Quartiere zu einem statistischen Stadtteil gehören, können die amtlichen Daten nicht separat für die beiden Quartiere ausgewiesen werden. Zudem können die Informationen nicht mit den offiziellen Arbeitslosenquoten verglichen werden, da die Anteile auf alle Befragten berechnet wurden, also auch Nichterwerbspersonen einschließen.
} 
anspruchsvollere Bausubstanz (insbesondere die umgenutzte Textilfabrik mit Lofts), eine vielfältigere gastronomische Infrastruktur sowie eine größere Nähe zu Innenstadt und Grünflächen aufweist.

In den beiden ausgewählten Quartieren wurden 990 Anschreiben im Vorfeld der ersten Befragungswelle in alle Briefkästen mit einem beschrifteten Klingelschild verteilt, die die eigentliche Befragung ankündigte. Es wurden also alle Haushalte im Quartier kontaktiert. Die zweite Befragungswelle im Jahr 2005 wurde in gleicher Weise durchgeführt. Bei der eigentlichen Befragung wurden die Haushalte persönlich kontaktiert und um Teilnahme an der Befragung gebeten (vgl. für die Durchführung derartiger Befragungen: Buzar et al. 2007). Darüber hinaus wurde ein Termin für die Abholung des Fragebogens vereinbart. Die eigentliche Befragung fand also als schriftliche Befragung statt, es wurde im Bedarfsfall allerdings Unterstützung durch die Interviewer angeboten. Innerhalb der Haushalte wurde nach der Geburtstagsmethode ein erwachsenes Haushaltsmitglied für die Befragung ausgewählt. Bei der ersten Welle der Befragung belief sich der Rücklauf auf 372 Fragebögen (37,6 \%), bei der zweiten Welle auf 347 Fragebögen $(35,1 \%) .{ }^{4}$ Personen, die bei beiden Wellen befragt wurden, werden in den folgenden Analysen jeweils nur einmal berücksichtigt. 5

Auf der Grundlage der schriftlichen Befragung können die zentralen Konstrukte der theoretischen Diskussion in Abschnitt 2 systematisch operationalisiert werden. Im ersten Schritt sollen nun die abhängigen Variablen betrachtet werden. Die zentrale abhängige Variable für die Wohnstandortwahl ist das Quartier, in dem eine Person wohnt. Wie in der Skizze deutlich wurde, handelt es sich hier tatsächlich um zwei Quartiere, die sich durch lebensstilrelevante Merkmale unterscheiden und damit den in Abschnitt 2 skizzierten Bedingungen genügen. Darüber hinaus kann die Wohnstandortwahl auch durch eine zweite Variable operationalisiert werden und zwar durch die Angabe, ob eine Person in einer Loftwohnung lebt oder nicht. Bei einem Loft handelt es sich zwar um einen spezifischen Typ von Wohnung, da dieser sich aber in bestimmten Gebäuden befindet, ist damit auch eine Auswahl des Wohnstandortes verbunden. Bei der Operationalisierung der Wohnungsmerkmale selbst orientiert sich die Studie an den zentralen Eigenschaften, die in der Forschung über Reurbanisierung und Gentrifizierung immer wieder genannt werden

\footnotetext{
${ }^{4}$ Die genannten Ausschöpfungsquoten sind allerdings nur als Untergrenzen zu verstehen. Da nicht jede Wohnung mit einem beschrifteten Klingelschild auch tatsächlich bewohnt ist, kann die Stichprobe nicht exakt definiert werden.

${ }^{5}$ In der zweiten Welle der Befragung konnten die Teilnehmer angeben, ob sie auch schon bei der ersten Welle einen Fragebogen beantwortet hatten. Diese Angabe erlaubte die Identifikation von Befragten, die an beiden Wellen teilgenommen haben.
} 
(Blasius 1993; Blasius und Friedrichs 2011), also der Größe der Wohnungen, ihrer Ausstattung mit Holzfußböden und Stuckdecken. Darüber hinaus wurde noch berücksichtigt, ob die Wohnung über einen Balkon verfügt. Bei den drei Ausstattungsmerkmalen handelt es sich um dichotome Variablen, dagegen handelt es sich bei der Größe der Wohnung (in qm pro Kopf) um eine intervallskalierte Größe. Schließlich sollen auch die Freiheitsgrade der Befragten bei der Auswahl ihrer Wohnung betrachtet werden, um das Ausmaß einer präferenzgesteuerten Entscheidung zu bestimmen. Dazu werden die Kriterien betrachtet, die bei der Wohnungssuche nach Auskunft der Befragten zu Grunde gelegt wurden. Eher enge Freiheitsgrade werden durch das Preiskriterium operationalisiert (Die Wohnung wurde ausgewählt, weil sie preisgünstig war). Dagegen können größere Freiheitsgrade durch eher hedonistische und ästhetische Merkmale operationalisiert werden (Die Wohnung gefällt, die Umgebung der Wohnung gefällt, die Wohnung liegt in Innenstadtnähe). In allen vier Fällen handelt es sich um dichotome Variablen.

In der theoretischen Diskussion in Abschnitt 2 wurde deutlich gemacht, dass die statistische Relevanz von individuellen Merkmalen wie Lebensstilen und sozioökonomischer Position immer davon abhängig ist, dass auch das Wohnungsangebot in entsprechender Hinsicht differenziert ist. Im Hinblick auf die Lebensstile wurde schon erläutert, dass die betrachteten Wohnstandorte und Wohnungsmerkmale als durchaus lebensstilrelevant betrachtet werden können. Im nächsten Schritt soll kurz skizziert werden, dass dies auch für die ökonomische Ausstattung der befragten Haushalte gilt. Dazu kann auf zwei Informationsquellen zurückgegriffen werden. Erstens wurden die Haushalte in der zweiten Welle der Befragung auch nach ihrer Warmmiete befragt, so dass analysiert werden konnte, ob bestimmte Wohnungsmerkmale und Standorte für die Miete pro Quadratmeter relevant sind. Zweitens wurden vom Juli 2002 bis zum Juni 2003 Mietwohnungsangebote für die beiden Quartiere im Internet einer Inhaltsanalyse unterzogen. Dabei wurden alle Wohnungsannoncen für die beiden Gebiete erhoben und Informationen über die Lage, den Preis und die Ausstattung der angebotenen Mietobjekte erhoben. So konnte eine kleine Datenbank generiert werden, die ebenfalls eine Analyse der Determinanten der Miete pro Quadratmeter erlaubt. Im Hinblick auf die betrachteten Wohnstandort- und Wohnungsmerkmale weisen die statistischen Analysen dieser Datenquellen übereinstimmend darauf hin (siehe Tabelle $1 \mathrm{im}$ Anhang), dass Wohnungen im Quartier mit Aufwertungstendenzen eine höhere Miete pro Quadratmeter aufweisen als Wohnungen im Vergleichsquartier. Auch für den Balkon und den Holzfußboden als Ausstattungsmerkmale zeigen beide Analysen übereinstimmend, dass diese Merkmale zu einer höheren Miete führen. Dagegen kann nur mit Hilfe der Befragungsdaten 
gezeigt werden, dass Loftwohnungen typischerweise höhere Mieten aufweisen und dass Stuckdecken als Ausstattungsmerkmal keinen statistisch signifikanten Einfluss haben. Darüber hinaus kann generell unterstellt werden, dass der Gesamtmietzins mit der Größe der Wohnung ansteigt, die Analyse der Wohnungsangebote weist allerdings mit zunehmender Quadratmeterzahl auf einen sinkenden Quadratmeterpreis hin, während sich dieses Ergebnis in den Befragungsdaten nicht zeigt. Generell kann aber festgehalten werden, dass für die Wohnstandort- und Wohnungsmerkmale als abhängige Variablen demonstriert werden konnte, dass diese, mit Ausnahme von Stuckdecken, mit deutlichen Differenzen im Mietpreis einer Wohnung einhergehen, so dass auch die ökonomische Ausstattung der Befragten für die Auswahl von Wohnungen mit diesen Merkmalen relevant sein sollte. Sowohl die Wohnstandorte als auch die Wohnungsmerkmale weisen also lebensstilrelevante Differenzierungen und entscheidungsrelevante Unterschiede in den ökonomischen Kosten auf, so dass die im theoretischen Modell genannten Bedingungen für die Relevanz sowohl des ersten als auch des zweiten Filters im Entscheidungsprozess gegeben sind.

Im nächsten Schritt soll nun die Operationalisierung der erklärenden Variablen betrachtet werden. Die für die Wohnstandortwahl relevanten Eigenschaften der Lebensstile wurden entsprechend den zwei in Abschnitt 2 skizzierten Mechanismen durch zwei Variablen operationalisiert. Bezugnehmend auf die Diskussion über die typischen Vorlieben von Personengruppen, die in innenstadtnahe Wohnlagen ziehen, wurde erstens eine additive Skala aus Aktivitäten gebildet, die typischerweise in städtischen Räumen konzentriert sind (Kino, Kneipe, Restaurant, Oper, Konzerthaus, Kunstmuseum, Theater - alle Items wurden auf einer fünfstufigen Skala abgefragt). Diese Skala wird hier als urbane Kultur bezeichnet (Cronbachs Alpha: 0,75). Zweitens wurde eine Hochkulturskala gebildet, da in den theoretischen Überlegungen über Gentrifizierung auch immer wieder darauf verwiesen wurde, dass mit einer hochkulturellen Orientierung eine stärkere Ästhetisierung auch von architektonischen Merkmalen und der Wohnungsausstattung verbunden ist. Diese Hochkulturskala wurde additiv aus der Vorliebe für Opernmusik und klassische Musik (fünfstufige Skala) gebildet (Cronbachs Alpha: 0,79). Da sich in der Forschung zur Wohnstandortwahl immer wieder gezeigt hat, dass die Lebensform eine zentrale Rolle spielt, wurde in einer dichotomen Variable erfasst, ob ein Befragter mit einem Partner zusammenlebt und mit einer weiteren dichotomen Variable, ob im Haushalt der Person auch Kinder leben. Das Einkommen der Befragten wurde als bedarfsgewichtetes Äquivalenzeinkommen nach der neuen OECD-Skala gebildet. Angesichts der deutlichen Mietpreisdifferenzen zwischen Wohnungen mit unterschiedlichen Ausstattungsmerkmalen ist das Einkommen eine zentrale Determinante der 
Opportunitätsstrukturen einer Person auf dem Wohnungsmarkt. Darüber hinaus wurden Alter, Geschlecht und Bildung als Kontrollvariablen berücksichtigt. Auf dieser Datengrundlage können nun die theoretisch begründeten Fragestellungen aus dem vorhergehenden Abschnitt empirisch untersucht werden.

\section{Empirische Resultate}

Im vorhergehenden Abschnitt wurden die Erhebung von Befragungsdaten im Rahmen einer Quartiersstudie und die Operationalisierung der theoretischen Konstrukte auf dieser Datenbasis erläutert. Auf dieser Grundlage kann nun zur Untersuchung der Forschungsfragen übergegangen werden: erstens der Prüfung des vermuteten Einflusses der Lebensstile auf die Wohnstandortwahl und die Wohnungsmerkmale sowie zweitens der Analyse der Determinanten einer präferenzgesteuerten Wohnstandortwahl durch sozialstrukturelle Merkmale und Lebensstile.

In einem ersten Schritt soll die Relevanz der Lebensstile und der ökonomischen Restriktionen für die Wohnstandortwahl betrachtet werden. Hier werden als abhängige Variablen einerseits die Auswahl einer Wohnung im Quartier mit Aufwertungstendenz und andererseits die Auswahl einer Loftwohnung betrachtet. Da es sich hier um dichotome Variablen handelt, wurden logistische Regressionen berechnet, deren Ergebnisse in Tabelle 1 präsentiert werden. Das Wohnen im Quartier mit Aufwertungstendenz wird offensichtlich durch Determinanten aus allen betrachteten Variablengruppen geprägt. Erstens zeigt sich, dass in dieses innenstadtnähere und baulich attraktivere Quartier, das ein größeres Angebot an Freizeitmöglichkeiten aufweist, tatsächlich eher Personen mit einer Hochkulturorientierung und einer urbanen Kulturorientierung ziehen. Darüber hinaus wird dieses Quartier aber auch von Personen mit Partnern, mit Kindern und von älteren Mietern vorgezogen. Schließlich hat auch das Einkommen einen starken Einfluss auf die Wohnstandortentscheidung. Die Ergebnisse für das Wohnen im Loft sind weniger vielfältig: hier hat eine urbane Kulturorientierung nur noch einen auf dem $10 \%$ Niveau statistisch signifikanten Einfluss, während sowohl das Einkommen als auch das Wohnen mit dem Partner für das Leben in einer solchen Wohnform relevant ist. Damit zeigt sich, dass die Lebensstile der befragten Personen durchaus einen Einfluss auf ihre Wohnstandortwahl haben, dieser ist aber für die Auswahl des Quartiers ausgeprägter als für das Wohnen im Loft. Insgesamt zeigt sich in diesen Resultaten auch, dass wir es in dem Quartier mit Aufwertungstendenz und in den Lofts nicht mit sogenannten Pionieren zu tun haben, sondern mit einer Personengruppe, die eher den sogenannten Gentrifiern zugerechnet werden kann, die also einen Partner haben, zum Teil 
Kinder und über überdurchschnittliche Einkommen verfügen. Dies ist nicht zuletzt auf die eher anspruchsvolle Sanierung der Gebäude in diesem Quartier zurückzuführen, wie dies insbesondere am Beispiel der Loftwohnungen in einem umgenutzten Fabrikgebäude demonstriert wurde (siehe auch Büttner und Mühmer 2004). ${ }^{6}$

Tabelle 1: Determinanten der Wohnstandortwahl

\begin{tabular}{|l|l|l|}
\hline & Quartier & Loftwohnung \\
\hline Hochkulturorientierung & $1,32^{* *}$ & 1,06 \\
& 7,66 & 0,12 \\
\hline Urbane Kultur & $3,58^{* *}$ & $2,44^{+}$ \\
& 17,65 & 3,81 \\
\hline Geschlecht & 0,72 & 0,73 \\
Referenz: weiblich & 2,65 & 1,01 \\
\hline Alter & $1,02^{* *}$ & 1,00 \\
& 10,30 & 0,12 \\
\hline Partner & $2,14 * *$ & $2,03 *$ \\
& 13,42 & 4,73 \\
\hline Kinder & $2,00^{* *}$ & 0,54 \\
& 7,15 & 1,89 \\
\hline Bildung & 0,95 & 1,09 \\
& 0,20 & 0,20 \\
\hline Einkommen & $3,84^{* *}$ & $2,88^{* *}$ \\
& 42,97 & 29,49 \\
\hline Pseudo-R & 0,35 & 0,23 \\
\hline
\end{tabular}

Logistische Regressionsmodelle. Angegeben sind die entlogarithmierten Regressionskoeffizienten sowie die Waldstatistiken. $+\mathrm{p}<0,1 ; * \mathrm{p}<0,05 ; * * \mathrm{p}<0,01$

Im zweiten Schritt soll nun der Einfluss der Lebensstile und der ökonomischen Restriktionen auf die Merkmale der ausgewählten Wohnungen betrachtet werden. Im Anschluss an die

\footnotetext{
${ }^{6}$ Im Rahmen des Lehrforschungsprojektes wurde auch eine Befragung von Bewohnern umgenutzter Industriegebäude in angrenzenden Stadtteilen durchgeführt, insbesondere einer ehemaligen Spinnerei, die vor allem Künstlern Wohn- und Arbeitsraum bietet und weniger aufwändig saniert wurde. Dabei wurde deutlich, dass diese Personen eher den Merkmalen der Pioniere entsprechen (geringeres Alter, niedrigeres Einkommen). Damit wird deutlich, dass die Merkmale der Gebäudebewohner typischerweise auch von den Anbietern und ihren Investitionen in ein bestimmtes Sanierungsniveau gesteuert werden.
} 
Ergebnisse von Blasius (1993) wird hier der Fokus insbesondere auf die Größe des Wohnraums, die Ausstattung mit Holzfußböden und mit Stuckdecken gelegt. Darüber hinaus soll aber auch betrachtet werden, ob die Wohnungen der Befragten einen Balkon aufweisen. Um den Einfluss der Lebensstile und der ökonomischen Restriktionen unter Kontrolle von weiteren Determinanten zu analysieren wurden für die Merkmale Holzböden ${ }^{7}$, Balkon und Stuckdecke logistische Regressionen durchgeführt, da es sich hier um dichotome Variablen handelt. Dagegen wurde für den Wohnraum (pro Kopf) eine lineare Regression berechnet. Die Resultate für alle vier Modelle werden in Tabelle 2 präsentiert. Betrachtet man den Einfluss der Lebensstile auf die vier abhängigen Variablen, so ist dieser als eher schwach ausgeprägt zu bezeichnen. Die Hochkulturorientierung ist in keinem Fall relevant, die urbane Kulturorientierung hat einen, auf dem $10 \%$ Niveau signifikanten, positiven Einfluss auf die Größe der Wohnung, die Ausstattung mit einem Balkon und einen signifikanten positiven Effekt auf das Vorhandensein einer Stuckdecke in der Wohnung. Diese Resultate entsprechen den Thesen aus der Literatur über die Wohnungsvorlieben urban orientierter Bevölkerungsgruppen, die Zusammenhänge sind aber eher schwach. Betrachtet man die demographischen Merkmale, so stellt man auch hier nur wenige statistisch signifikante Zusammenhänge fest: Männer geben etwas häufiger an eine Stuckdecke in der Wohnung zu haben, während Personen mit höherem Alter tendenziell eher eine größere Wohnung und einen Balkon als Ausstattungsmerkmal aufweisen. Analysiert man die Lebensform als Einflussgröße, so zeigt sich, dass Partnerhaushalte und Haushalte mit Kindern weniger Wohnraum pro Kopf zur Verfügung haben, Partnerhaushalte haben häufiger einen Balkon, Haushalte mit Kindern verfügen über alle betrachteten Ausstattungsmerkmale häufiger. ${ }^{8}$ Dies deutet insgesamt darauf hin, dass der Bereich des Wohnens für Paare und Familien eine besonders hohe Relevanz aufweist, so dass eher Wohnungen mit einer anspruchsvolleren Ausstattung ausgewählt werden. Für die sozialstrukturellen Merkmale zeigt sich wiederum für die Bildung kein statistisch signifikanter Effekt, während das Einkommen erwartungsgemäß für die Größe der angemieteten Wohnung sowie auch für die Ausstattung mit Holzfußboden und Balkon relevant ist. Entsprechend der in Abschnitt 3 formulierten Erwartungen wird kein statistisch signifikanter Effekt des Einkommens auf das Vorhandensein einer Stuckdecke in der Wohnung festgestellt. Insgesamt zeigt sich aber in diesen Modellen, dass vor allem das

\footnotetext{
${ }^{7}$ Für das Merkmal Holzfußboden liegen deutlich weniger Fälle vor als für die Vergleichsmerkmale, da diese Variable nur in der zweiten Welle der Befragung erhoben wurde.

${ }^{8}$ Etwas überraschend mag sein, dass Familienhaushalte häufiger in Wohnungen mit Stuckdecken leben. Dies hat seinen Hintergrund in der Tatsache, dass größere Wohnungen eher Stuckdecken aufweisen und Familien eher in größeren Wohnungen leben.
} 
Einkommen und die Lebensform das Leben in qualitativ hochwertig ausgestatten und großen Wohnungen prägt, weniger dagegen der Lebensstil.

Tabelle 2: Determinanten der Wohnungsauswahl

\begin{tabular}{|c|c|c|c|c|}
\hline & Wohnraum & Holzböden & Balkon & Stuckdecke \\
\hline \multirow[t]{2}{*}{ Hochkulturorientierung } & 0,02 & 1,022 & 0,96 & 1,24 \\
\hline & 0,61 & 2,25 & 0,22 & 1,26 \\
\hline \multirow[t]{2}{*}{ Urbane Kultur } & $0,07^{+}$ & 1,29 & $1,73^{+}$ & $3,62 *$ \\
\hline & 1,69 & 0,44 & 3,69 & 5,20 \\
\hline Geschlecht & 0,01 & 1,05 & 0,86 & $1,83^{+}$ \\
\hline Referenz: weiblich & 0,25 & 0,04 & 0,65 & 2,77 \\
\hline \multirow[t]{2}{*}{ Alter } & $0,19 * *$ & 0,99 & $1,03 * *$ & 1,00 \\
\hline & 4,91 & 1,27 & 12,61 & 0,10 \\
\hline \multirow[t]{2}{*}{ Partner } & $-0,37 * *$ & 1,11 & $2,16 * *$ & 1,92 \\
\hline & $-10,67$ & 0,16 & 15,30 & 2,68 \\
\hline \multirow[t]{2}{*}{ Kinder } & $-0,23 * *$ & $2,41 *$ & $3,00 * *$ & $3,64 * *$ \\
\hline & $-6,74$ & 5,79 & 16,49 & 10,21 \\
\hline \multirow[t]{2}{*}{ Bildung } & $-0,01$ & 1,11 & 0,89 & 1.16 \\
\hline & $-0,36$ & 0,39 & 1,13 & 0,42 \\
\hline \multirow[t]{2}{*}{ Einkommen } & $0,37 * *$ & $3,93 * *$ & $2,29 * *$ & 1,09 \\
\hline & 10,29 & 24,64 & 22,64 & 0,11 \\
\hline Korrigiertes & 0,41 & 0,23 & 0,22 & 0,15 \\
\hline \multicolumn{5}{|l|}{ Pseudo-R ${ }^{2}$} \\
\hline $\mathrm{N}$ & 548 & 324 & 595 & 597 \\
\hline
\end{tabular}

Logistische Regressionsmodelle in Spalten 2 bis 4, lineares Regressionsmodell in Spalte 1. Angegeben sind für die logistischen Regressionsmodelle die entlogarithmierten Regressionskoeffizienten sowie die Waldstatistiken. Für das lineare Regressionsmodell sind der standardisierte Regressionskoeffizient sowie der t-Wert angegeben. $+\mathrm{p}<0,1 ; * \mathrm{p}<0,05 ; * * \mathrm{p}<0,01$

Zum Abschluss soll nun das Ausmaß der subjektiv wahrgenommenen Präferenzsteuerung bei der Wohnungsauswahl und seine Prägung durch sozialstrukturelle Merkmale untersucht werden. Dafür wird betrachtet, ob die Befragten die folgenden vier Kriterien als relevant für ihre Wohnstandortwahl angegeben haben: erstens günstiger Preis der Wohnung, zweitens ästhetisches Wohlgefallen an der Wohnung, drittens ästhetisches Wohlgefallen an der 
Umgebung und viertens die Innenstadtnähe der Wohnung ${ }^{9}$. Da alle vier Variablen dichotom codiert sind, wurden vier logistische Regressionen berechnet, um die Prägung dieser Kriterien durch sozialstrukturelle Merkmale zu analysieren. Die Ergebnisse der Analysen werden in Tabelle 3 präsentiert. Betrachtet man zuerst die Relevanz der Lebensstile, so zeigt sich, dass diese nur für diejenigen Kriterien relevant sind, die einen ästhetischen Bezug aufweisen und auf einen größeren Handlungsspielraum der Befragten hinweisen. Während die Hochkulturorientierung allerdings nur einen schwach signifikanten Effekt auf das ästhetische Wohlgefallen an der Wohnumgebung hat, ist die urbane Kulturorientierung für die ästhetische Bewertung der Wohnung und der Umgebung prägend und darüber hinaus auch für die Nennung des Kriteriums Innenstadtnähe. Hier zeigt sich sehr deutlich, dass Personen mit einer urbanen Kulturorientierung nicht nur ihre Wohnung und die unmittelbare Wohnumgebung unter ästhetischen Gesichtspunkten bewerten und auswählen, sondern auch auf die typischerweise in der Innenstadt gelegenen Möglichkeiten zum Ausleben ihrer kulturellen Vorlieben achten. Die demographischen Merkmale haben in diesen vier Modellen einen eher geringeren Einfluss: Männer nennen etwas häufiger als Frauen das Preiskriterium für die Wohnungsauswahl, während ältere Menschen seltener auf das Preiskriterium und eine ästhetische Beurteilung der Wohnumgebung verweisen, wobei letzteres allerdings nur auf dem $10 \%$ Niveau signifikant ist. Die Lebensform erweist sich dagegen wiederum als zentral für die Auswahlkriterien: Sowohl Personen in Partnerhaushalten als auch Personen mit Kindern geben seltener das Preiskriterium an, dagegen häufiger die eher ästhetischen Beurteilungskriterien, allerdings mit Ausnahme der Innenstadtnähe. Schließlich erweist sich das Einkommen als zentrale Determinante der Nennung von bestimmten Auswahlkriterien. Das Preiskriterium wird ganz überwiegend von Personen mit niedrigem Einkommen genannt, während die Verwendung der eher ästhetischen Kriterien von einem höheren Einkommen ermöglicht wird. Damit zeigen diese Analysen sehr deutlich, dass die Auswahl von Wohnstandorten und Wohnungen in der Regel durch die verfügbaren Ressourcen von Personen und damit durch den ersten Filter des oben skizzierten entscheidungstheoretischen Modells beschränkt ist: Personen mit sehr tiefem Einkommen haben quasi keine Wahl und müssen primär auf den Preis achten, während Personen mit mittleren und höheren Einkommen bei der Auswahl auch auf ästhetische Kriterien zurückgreifen können (vgl. Blasius und Friedrichs 2011).

\footnotetext{
${ }^{9}$ Diese Variable wurde nur in der zweiten Welle der Befragung erhoben, so dass deutlich weniger Fälle als für die anderen Auswahlkriterien vorliegen.
} 
Tabelle 3: Präferenzsteuerung der Wohnstandortwahl

\begin{tabular}{|c|c|c|c|c|}
\hline & $\begin{array}{l}\text { Wohnung } \\
\text { preisgünstig }\end{array}$ & $\begin{array}{l}\text { Schöne } \\
\text { Wohnung }\end{array}$ & $\begin{array}{l}\text { Schöne } \\
\text { Gegend }\end{array}$ & Innenstadt \\
\hline Hochkulturorientierung & $\begin{array}{l}0,90 \\
1,10\end{array}$ & $\begin{array}{l}1,15 \\
2,29\end{array}$ & $\begin{array}{l}1,19^{+} \\
0,63\end{array}$ & $\begin{array}{l}1,15 \\
1,11\end{array}$ \\
\hline Urbane Kultur & $\begin{array}{l}1,11 \\
0,13\end{array}$ & $\begin{array}{l}2,72 * * \\
12,91\end{array}$ & $\begin{array}{l}1,98^{*} \\
4,57\end{array}$ & $\begin{array}{l}3,97 * * \\
12,54\end{array}$ \\
\hline $\begin{array}{l}\text { Geschlecht } \\
\text { Referenz: weiblich }\end{array}$ & $\begin{array}{l}1,48^{*} \\
3,90\end{array}$ & $\begin{array}{l}0,98 \\
0,01\end{array}$ & $\begin{array}{l}1,13 \\
0,49\end{array}$ & $\begin{array}{l}0,87 \\
0,29\end{array}$ \\
\hline Alter & $\begin{array}{l}0,97 * * \\
18,30\end{array}$ & $\begin{array}{l}0,99 \\
2,32\end{array}$ & $\begin{array}{l}0,99^{+} \\
3,06\end{array}$ & $\begin{array}{l}0,99 \\
1,68\end{array}$ \\
\hline Partner & $\begin{array}{l}0,67 * \\
3,89\end{array}$ & $\begin{array}{l}2,01 * * \\
12,82\end{array}$ & $\begin{array}{l}1,43^{*} \\
3,92\end{array}$ & $\begin{array}{l}1,49 \\
2,20\end{array}$ \\
\hline Kinder & $\begin{array}{l}0,65^{+} \\
2,97\end{array}$ & $\begin{array}{l}0,92 \\
0,14\end{array}$ & $\begin{array}{l}1,61 * \\
4,55\end{array}$ & $\begin{array}{l}1,18 \\
0,26\end{array}$ \\
\hline Bildung & $\begin{array}{l}1,03 \\
0,07\end{array}$ & $\begin{array}{l}0,91 \\
0,90\end{array}$ & $\begin{array}{l}0,99 \\
0,00\end{array}$ & $\begin{array}{l}0,87 \\
0,76\end{array}$ \\
\hline Einkommen & $\begin{array}{l}0,25 * * \\
45,38\end{array}$ & $\begin{array}{l}2,14 * * \\
19,50\end{array}$ & $\begin{array}{l}1,10 \\
0,50\end{array}$ & $\begin{array}{l}1,67 * * \\
6,68\end{array}$ \\
\hline Pseudo- $^{2}$ & 0,27 & 0,18 & 0,07 & 0,18 \\
\hline $\mathrm{N}$ & 597 & 597 & 597 & 325 \\
\hline
\end{tabular}

Logistische Regressionsmodelle. Angegeben sind die entlogarithmierten Regressionskoeffizienten sowie die Waldstatistiken. $+\mathrm{p}<0,1 ; * \mathrm{p}<0,05 ; * * \mathrm{p}<0,01$

\section{Zusammenfassung und Diskussion}

In der Lebensstilforschung und verschiedenen Disziplinen der Raumforschung wird die These vertreten, dass Lebensstile relevant für Entscheidungen in unterschiedlichen Verhaltensbereichen, insbesondere aber im Feld der Auswahl von Wohnungen und Wohnstandorten sind. Dabei wird einerseits darauf verwiesen, dass Personen mit einem ausgesprochen urbanen Lebensstil, der auf die Nutzung typisch städtischer Kultur- und Freizeitangebote ausgerichtet ist, eine Präferenz für attraktive, innerstädtische gelegene Wohnungen haben. Andererseits wird unterstellt, dass Personen mit einer hochkulturellen Orientierung eine ästhetisierende Perspektive nicht nur auf Werke der legitimen Kultur haben, sondern auch auf Objekte und Gegenstände des Alltags, also auch ihrer Wohnumgebung. 
Daher muss angenommen werden, dass diese Personen auch ihre Wohnung und ihren Wohnstandort auf der Grundlage von ästhetischen Bewertungskriterien auswählen. Die Befundlage zu dieser Frage ist allerdings ausgesprochen uneinheitlich. Sehen manche Autoren Lebensstile als wichtige Erklärung für Wohnstandortwahlen und die Nutzung der räumlichen Infrastruktur an (Klee 2003; Scheiner 2006; Spellerberg 2007), so sehen andere Lebensstile als wenig erklärungskräftig in diesem Bereich (Spiegel 2000; Kauko 2006; Otte 2004; Heijs et al. 2009; Blasius und Friedrichs 2011).

Um diese uneinheitlichen Ergebnisse interpretieren $\mathrm{zu}$ können und eine systematischere theoretische Grundlage für die Prüfung des Einflusses von Lebensstilen auf die Wohnstandortwahl zu entwickeln, wurde ein entscheidungstheoretisches Modell vorgestellt, das in ähnlichen Varianten in der Stadtsoziologie (Friedrichs 1988, 2008) und der Lebensstilforschung (Rössel 2006a, 2008) verwendet wird, um Entscheidungen in unterschiedlichen Verhaltensbereichen zu erklären. Entscheidungen werden hier als das Resultat von zwei Filterprozessen erklärt, wobei der erste Filter auf der Grundlage der vorliegenden Alternativen und der Ressourcenausstattung von Personen die tatsächlich verfügbaren Optionen einer Person bestimmt und der zweite Filter aus den Präferenzen von Akteuren für bestimmte Eigenschaften dieser Alternativen besteht. Auf der Grundlage dieses Modells konnten Bedingungen abgeleitet werden, unter denen Lebensstile sich als relevant für die Auswahl von Wohnungen bzw. Wohnstandorten erweisen sollten. Erstens müssen Handlungsalternativen betrachtet werden, die sich tatsächlich in lebensstilrelevanter Weise unterscheiden. Zweitens müssen die Lebensstile als dimensionale Variablen konstruiert werden, so dass diese tatsächlich als Präferenzen für bestimmte Merkmale von Wohnungen bzw. Wohnstandorten interpretiert werden können. Drittens kann geschlussfolgert werden, dass vor allem Personen mit einer gehobenen Ressourcenausstattung, für die also der erste Entscheidungsfilter wenig relevant ist, bei einer Entscheidung in starkem Maße präferenzgesteuert vorgehen können.

Bei der Durchführung der empirischen Studie wurden die vorstehenden Punkte systematisch berücksichtigt, so dass ausgeprägte statistische Effekte der ausgewählten Lebensstilvariablen (Urbane Kulturorientierung, Hochkulturorientierung) auf die Auswahl von Wohnungen bzw. Wohnstandorten erwartet werden konnten. Diese Erwartung wurde noch durch die Tatsache verstärkt, dass es sich hier um eine Studie über den ostdeutschen Wohnungsmarkt handelt, der durch einen ausgesprochenen Angebotsüberhang charakterisiert ist und damit für eine präferenzgesteuerte Wohnungsauswahl günstige Bedingungen bietet. Die empirischen Resultate der Studie, die auf einer Quartiersbefragung im Leipziger Westen basiert, fallen 
allerdings nicht so eindeutig aus. Von den beiden Lebensstildimensionen (Urbane Kulturorientierung, Hochkulturorientierung) zeigen sich für die Hochkulturorientierung lediglich zwei statistisch signifikante Effekte. Erstens ziehen hochkulturell orientierte Personen eher in das betrachtete Quartier mit Aufwertungstendenz und zweitens haben sie bei der Umzugsentscheidung die Attraktivität des Quartiers in besonderem Maße berücksichtigt. Für alle anderen betrachteten Wohnungs- und Wohnstandortmerkmale hat sich die Hochkulturorientierung als nicht relevant erwiesen. Die urbane Kulturorientierung hat sich in größerem Umfang als bedeutsam erwiesen. Insbesondere ein Einzug in das Quartier mit Aufwertungstendenz wurde deutlich durch diese Orientierung geprägt. Darüber hinaus zeigten sich auch Einflüsse auf das Wohnen in einem Loft, den verfügbaren Wohnraum pro Kopf sowie die Ausstattung der Wohnung mit einem Balkon und Stuckdecken. Dies sind typische Merkmale von Wohnungen, die auch in der Literatur über Gentrifizierung häufig genannt werden. Allerdings muss an dieser Stelle festgehalten werden, dass die urbane Kulturorientierung sich für diese Merkmale nicht als überaus bedeutsam herausgestellt hat, mehrere Effekte stellten sich als nur auf dem $10 \%$ Niveau statistisch signifikant dar. Bedeutsamer ist die urbane Kulturorientierung von Personen für die Kriterien der Wohnstandortwahl: hier zeigt sich, dass Akteure mit dieser Lebensstilausprägung eher ästhetische Kriterien bei der Auswahl von Wohnungen anlegen.

Zwei andere Variablen haben sich als durchgängig ausgesprochen relevant für die Auswahl von Wohnungen respektive Wohnstandorten erwiesen. Erstens das Einkommen, das in starkem Maße darüber bestimmt welche Wohnungen einer Person überhaupt zugänglich sind. Für alle Wohnungs- und Wohnstandortmerkmale, die mit klaren Preisdifferenzen verbunden waren, zeigten sich für das Einkommen als erklärende Variable hochsignifikante Einflüsse. Dies verdeutlicht die Relevanz des ersten Filters des entscheidungstheoretischen Modells. Dies wurde auch bei der Analyse des Ausmaßes von Präferenzsteuerung bei der Auswahl von Wohnungen ersichtlich: Personen mit niedrigen Einkommen mussten vor allem auf den Mietpreis achten, während Personen mit höherem Einkommen auch ästhetische Kriterien an die Wohnungen anlegen konnten. Dies verdeutlicht, dass der erste Filter des entscheidungstheoretischen Modells den Anwendungsbereich und die Relevanz des zweiten Filters stark strukturiert, wie dies auf der Grundlage des entscheidungstheoretischen Modells auch prognostiziert wurde (Friedrichs 1998; Blasius und Friedrichs 2011; Rössel 2006a, 2008). Es sind damit vor allem ressourcenstarke Personengruppen, die auf der Basis subjektiver Präferenzen ihre Wohnungen und Wohnstandorte auswählen können. Zweitens zeigt sich auch die Lebensform als überaus wichtig: Befragte mit Partnern bzw. mit Kindern 
unterschieden sich in ihrer Auswahl von Wohnungen und Wohnstandorten ausgeprägt von Singles. Dies bestätigt die Ergebnisse von Spellerberg (2007), die auf die Lebensform als wichtigste Determinante der Wohnstandortwahl hingewiesen hat.

Bei der Interpretation der empirischen Ergebnisse stellt sich allerdings die zentrale Frage, ob aus den vorliegenden Querschnittsdaten überhaupt Rückschlüsse auf die Richtung der Kausalität gezogen werden können. Dies ist grundsätzlich nicht möglich: die Ergebnisse können sowohl dafür sprechen, dass Personen mit bestimmten Lebensstilen sich bestimmte Wohnstandorte auswählen, aber auch dafür, dass Personen an bestimmten Wohnstandorten gewisse Lebensstile entwickeln. Es sollen an dieser Stelle zwei Argumente für die erste Position ins Feld geführt werden. Erstens wurden die Modelle auch unter Ausschluss aller Befragten berechnet, die schon länger als fünf Jahre in ihrer Wohnung leben. Die Ergebnisse haben sich nicht substantiell von den hier präsentierten empirischen Resultaten unterschieden. Da die Prägung von Lebensstilen durch die räumliche Umgebung, wenn überhaupt, nur nach zeitlicher Verzögerung einsetzt, sprechen diese Ergebnisse eher für die ursächliche Wirkung der Lebensstile als umgekehrt. Zweitens zeigen darüber hinaus die Analysen einer neueren Studie von Spellerberg (2011), dass Lebensstile primär durch die sozialstrukturelle Position einer Person geprägt werden und nur geringfügig durch den sozialräumlichen Kontext, wobei sie nach Umzügen praktisch keinen Einfluss des räumlichen Kontextes auf die Lebensstile feststellen konnte. Dies spricht zusammen genommen dafür, dass es sich bei den betrachteten Effekten um einen Einfluss der Lebensstile auf die Wohnstandortwahl und nicht umgekehrt handelt. Abschließend kann dies aber nur auf der Basis von Längsschnittdaten geprüft werden.

Zusammenfassend kann aber an dieser Stelle festgehalten werden, dass die vorliegende Studie eher eine skeptische Perspektive auf die Relevanz von Lebensstilen für die Auswahl von Wohnungen und Wohnstandorten stützt. Obwohl es sich hier um ein Studiendesign und einen Kontext gehandelt hat, in dem ein deutlicher Einfluss von Lebensstilen hätte sichtbar werden können, bleibt dieser insgesamt doch sehr beschränkt. Lediglich bei der Wohnstandortwahl im engeren Sinne zeigte sich ein deutlicher Effekt, während bei den Wohnungseigenschaften eher schwächere Effekte auftraten, wobei diese fast ausschließlich für die Lebensstildimension der urbanen Kulturorientierung sichtbar waren. Dagegen zeigten sich deutliche Einflüsse der Ressourcenausstattung von Personen und ihrer Lebensform auf die Auswahl von Wohnungen und Wohnstandorten. Insofern wird man damit schließen müssen, dass Lebensstile nur eine Variable unter anderen sind, die Wohnstandortentscheidungen beeinflussen und dies auch nur in einem wenig ausgeprägten Maß. Dies deckt sich allerdings 
mit den Erwartungen, die man auf der Grundlage des skizzierten entscheidungstheoretischen Modells formulieren kann. Da das Wohnungsangebot stark in seinen Kosten differiert, sollte man davon ausgehen, dass der erste Entscheidungsfilter in diesem Fall dominant ist, während der zweite Filter nur einen beschränkten Einfluss auf die endgültige Entscheidung haben sollte (Friedrichs 1998; Blasius und Friedrichs 2011; Rössel 2006a, 2008). Damit unterstützen die Ergebnisse der empirischen Studie die skizzierte entscheidungstheoretische Perspektive auf die Relevanz von Lebensstilen.

\section{Literatur}

Abel, Thomas, Andrea Abraham, und Kathrin Sommerhalder. 2006. Kulturelles Kapital, kollektive Lebensstile und die soziale Reproduktion gesundheitlicher Ungleichheit. In Gesundheitliche Ungleichheit, Hrsg. Matthias Richter und Klaus Hurrelmann, 185-198. Wiesbaden: VS Verlag für Sozialwissenschaften.

Ærø, Thorkild. 2006. Residential Choice from a Lifestyle Perspective. Housing, Theory and Society 23:109-230.

Arranz-Becker, Oliver, und Daniel Lois . 2010. Selection, Alignment and their Interplay. Origins of Lifestyle Homogamy in Couple Relationships. Journal of Marriage and the Family 72:1234-1248.

Beck, Ulrich. 1986. Risikogesellschaft. Auf dem Weg in eine andere Moderne. Frankfurt: Suhrkamp.

Berger, Peter A. 1986. Entstrukturierte Klassengesellschaft? Klassenbildung und Strukturen sozialer Ungleichheit im historischen Wandel. Opladen: Westdeutscher Verlag.

Blasius, Jörg. 1993. Gentrification und Lebensstile. Eine empirische Untersuchung. Wiesbaden: DUV.

Blasius, Jörg, und Jürgen Friedrichs. 2008. Lifestyles in Distressed Neighborhoods. A Test of Bourdieu's “Taste of Necessity“ Hypothesis. Poetics 36:24-44.

Blasius, Jörg, und Jürgen Friedrichs. 2011. Die Bedeutung von Lebensstilen für die Erklärung von sozial-räumlichen Prozessen. Erscheint in Lebensstilforschung (Sonderheft 51 der Kölner Zeitschrift für Soziologie und Sozialpsychologie), Hrsg. Jörg Rössel und Gunnar Otte. Wiesbaden: VS Verlag für Sozialwissenschaften.

Bourdieu, Pierre. 1982. Die feinen Unterschiede. Kritik der gesellschaftlichen Urteilskraft. Frankfurt: Suhrkamp. 
Büttner, Kerstin, und Karsten Mühmer. 2004. Revitalisierung ehemals industriell genutzter Bausubstanz durch Lofts - Beispiele aus Berlin und Leipzig. Berichte zur deutschen Landeskunde 78:55-71.

Beauregard, Robert A. 1986. The Chaos and Complexity of Gentrification. In Gentrification of the City, Hrsg. Neil Smith und Peter Williams, 35-55. Boston: Unwin Hyman.

Bridge, Gary. 2001. Estate Agents as Interpreters of Economic and Cultural Capital: The Gentrification Premium in the Sydney Housing Market. International Journal of Urban and Regional Research 25:87-101.

Brun, Jacques, und Jeanne Fagnani. 1994. Lifestyles and Locational Choices - Trade-offs and Compromises: A Case Study of Middle-class Couples Living in the Ile-de-France Region. Urban Studies 31:921-934.

Buzar, Stefan, Philip Ogden, Ray Hall, Annegret Haase, Sirun Kabisch, und Annett Steinführer. 2007. Splintering Urban Populations: Emergent Landscapes of Reurbanisation in Four European Cities. Urban Studies 44:651-677.

Dangschat, Jens. 1994. Segregation - Lebensstile im Konflikt, soziale Ungleichheiten und räumliche Disparitäten. In Lebensstile in den Städten, Hrsg. Jörg Blasius, 426-445. Opladen: Leske + Budrich.

Dangschat, Jens, und Jörg Blasius (Hrsg.). 1990. Gentrification. Die Aufwertung innenstadtnaher Wohnviertel. Frankfurt: Campus.

Friedrichs, Jürgen. 1988. Makro- und mikrosoziologische Theorien der Segregation. In Soziologische Stadtforschung (Sonderheft 28 der Kölner Zeitschrift für Soziologie und Sozialpsychologie), Hrsg. ders., 56-77. Opladen: Westdeutscher Verlag.

Friedrichs, Jürgen. 1998. Gentrification. In Großstadt. Soziologische Stichworte, Hrsg. Hartmut Häussermann, 57-67. Opladen: Leske + Budrich.

Friedrichs, Jürgen. 2008. Ethnische Segregation. In Migration und Integration (Sonderheft 48 der Kölner Zeitschrift für Soziologie und Sozialpsychologie), Hrsg. Frank Kalter, 380-411. Wiesbaden: VS Verlag für Sozialwissenschaften.

Friedrichs, Jürgen, und Karl-Dieter Opp. 2002. Rational Behavior in Everyday Situations. European Sociological Review 18:401-415.

Glatter, Jan, und Winfried Killisch. 2004. Gentrification in innenstadtnahen Wohnquartieren ostdeutscher Städte - das Beispiel der Dresdner Äußeren Neustadt. Berichte zur deutschen Landeskunde 78:41-54.

Haan, Jos de, und Wilfred Uunk. 2001. Kulturelle Ähnlichkeiten zwischen Ehepaaren. Der Einfluss von Partnerwahl, Restriktionen und gegenseitiger Beeinflussung. In Partnerwahl und 
Heiratsmuster. Sozialstrukturelle Voraussetzungen der Liebe, Hrsg. Thomas Klein, 77-97. Opladen: Leske + Budrich.

Harth, Annette, Herlyn, Ulfert, und Gitta Scheller. 1998. Segregation in ostdeutschen Städten: Eine empirische Studie. Opladen: Leske + Budrich.

Hartman, Peter H. 1999. Lebensstilforschung. Darstellung, Kritik und Weiterentwicklung. Opladen: Leske + Budrich.

Heijs, Wim, Marleen Carton, Jos Smeets, und Angelique van Gemert. 2009. The Labyrinth of Life-Styles. Journal of Housing and the Built Environment 24:347-356.

Hill, André, und Karin Wiest. 2004. Gentrification in ostdeutschen Cityrandgebieten? Theoretische Überlegungen zum empirischen Forschungsstand. Berichte zur deutschen Landeskunde 78:25-39.

Hradil, Stefan. 1987. Sozialstrukturanalyse in einer fortgeschrittenen Gesellschaft. Opladen: Leske + Budrich

Kauko, Tom. 2006. Expressions of Housing Consumer Preferences: Proposition for a Research Agenda. Housing, Theory and Society 23:92-108.

Klee, Andreas. 2003. Lebensstile, Kultur und Raum. Anmerkungen zum Raumbezug soziokultureller Gesellschaftsformationen. Geographische Zeitschrift 91:63-74.

Klein, Thomas, Sven Schneider, und Hannelore Löwel. 2001. Bildung und Mortalität. Die Bedeutung gesundheitsrelevanter Aspekte des Lebensstils. Zeitschrift für Soziologie 30:384400.

Klocke, Andreas, und Detlev Lück. 2001. Lebensstile in der Familie. Ifb-Materialien 3-2001.

Ley, David. 2003. Artists, Aestheticisation and the Field of Gentrification. Urban Studies 40: 2527-2544.

Marshall, Richard (Hrsg.). 2001. Waterfronts in Post-Industrial Cities. London/New York: Spon Press.

Mochmann, Ingvill C., und Yasemin El-Menouar. 2005. Lifestyle Groups, Social Milieus and Party Preference in Eastern and Western Germany. Theoretical Considerations and Empirical Results. German Politics 4:417-437.

Ohnmacht, Timo, Konrad Götz, und Helmut Schad. 2009. Leisure Mobility Styles in Swiss Conurbations: Construction and Empirical Analysis. Transportation 36:243-265.

Otte, Gunnar. 1997. Lebensstile versus Klassen - welche Sozialstrukturkonzeption kann die individuelle Parteipräferenz besser erklären? In Soziale Ungleichheit. Neue Befunde zu Strukturen, Bewusstsein und Politik, Hrsg. Walter Müller, 303-346. Opladen: Leske + Budrich. 
Otte, Gunnar. 2004. Sozialstrukturanalyse mit Lebensstilen. Wiesbaden: VS Verlag für Sozialwissenschaften.

Otte, Gunnar. 2005. Hat die Lebensstilforschung eine Zukunft? Eine Auseinandersetzung mit aktuellen Bilanzierungsversuchen. Kölner Zeitschrift für Soziologie und Sozialpsychologie 57: $1-31$.

Podmore, Julie. 1998. (Re)reading the Loft Living Habitus in Montreals Inner City. International Journal of Urban and Regional Research 22: 283-302.

Rössel, Jörg. 2005. Plurale Sozialstrukturanalyse. Eine handlungstheoretische Rekonstruktion der Grundbegriffe der Sozialstrukturanalyse. Wiesbaden: VS Verlag für Sozialwissenschaften.

Rössel, Jörg. 2006a. Kostenstruktur und Ästhetisierung. Zur Erklärungskraft von Lebensstilen. Kölner Zeitschrift für Soziologie und Sozialpsychologie 58:453-467.

Rössel, Jörg. 2006b. Allesfresser im Kinosaal? Zur Übertragbarkeit des Konzepts der kulturellen Allesfresser auf Deutschland. Soziale Welt 57:259-272.

Rössel, Jörg. 2008. Conditions for the Explanatory Power of Life Styles. European Sociological Review 24:231-241.

Rössel, Jörg, und Michael Hölscher. 2004. Soziale Milieus in Gaststätten. Eine Beobachtung. Sociologus 54:173-203.

Scheiner, Joachim. 2006. Wohnen und Aktionsraum: Welche Rolle spielen Lebensstil, Lebenslage und Raumstruktur? Geographische Zeitschrift 94:43-62.

Schneider, Nicole, und Annette Spellerberg. 1999. Lebensstile, Wohnbedürfnisse und räumliche Mobilität. Opladen: Leske + Budrich.

Schulze, Gerhard. 1992. Die Erlebnisgesellschaft. Kultursoziologie der Gegenwart. Frankfurt: Campus.

Spellerberg, Annette. 1997. Lebensstile und Wohnverhältnisse. Discussion Paper FS-III 97403. Berlin: Wissenschaftszentrum.

Spellerberg, Annette. 2007. Lebensstile im sozialräumlichen Kontext: Wohnlage und Wunschlagen. In Lebensstile, Soziale Lagen und Siedlungsstrukturen, Hrsg. Jens S. Dangschat und Alexander Hamedinger, 182-204. Hannover: Verlag der ARL.

Spellerberg, Annette. 2011. Kultur in der Stadt - Autopflege auf dem Land? Eine Analyse sozialräumlicher Differenzierungen des Freizeitverhaltens auf der Basis des SOEP 1998 2008. Erscheint in Lebensstilforschung (Sonderheft 51 der Kölner Zeitschrift für Soziologie und Sozialpsychologie), Hrsg. Jörg Rössel und Gunnar Otte. Wiesbaden: VS Verlag für Sozialwissenschaften. 
Spiegel, Erika. 2000. Haushaltsformen und Lebensstile im Lebensverlauf - Wohn- und standortbedürfnisse und -präferenzen. In Stadt und soziale Ungleichheit, Hrsg. Annette Harth, Gitta Scheller, und Wulf Tessing, 197-216. Opladen: Leske + Budrich.

Steinführer, Annett. 2004. Wohnstandortentscheidungen und städtische Transformation. Vergleichende Fallstudien für Ostdeutschland und Tschechien. Wiesbaden: VS Verlag für Sozialwissenschaften.

Steinführer, Annett, und Annegret Haase. 2009. Flexible - Inflexible: Socio-Demographic, Spatial and Temporal Dimensions of Flat Sharing in Leipzig (Germany). Geojournal 74:567587.

Thomas, Dirk, Urs Fuhrer, und Claudia Quaiser-Pohl. 2008. Akteure der Gentrification und ihre Ortsbindung: Eine Studie in einem städtischen Sanierungsgebiet in Ostdeutschland. Kölner Zeitschrift für Soziologie und Sozialpsychologie 60:339-366.

Wiest, Karin, und André Hill. 2004a. Segregation und Gentrification in der schrumpfenden Stadt? Städte im Umbruch 1/04:62-66.

Wiest, Karin, und André Hill. 2004b. Sanfte Gentrifizierung, Studentifizierung und Inseln ethnischer Konzentration in ostdeutschen Innenstadtrandgebieten? Raumforschung und Raumordnung 62:361-374.

Wiest, Karin, und Romy Zischner. 2006. Aufwertung innerstädtischer Altbaugebiete in den neuen Bundesländern - Prozesse und Entwicklungspfade in Leipzig. Deutsche Zeitschrift für Kommunalwissenschaften Heft 1:99-121.

Zukin, Sharon. 1989. Loft Living. New Brunswick: Rutgers University Press.

Zukin, Sharon. 1998. Urban Lifestyles: Diversity and Standardisation in Spaces of Consumption. Urban Studies 35:825-839. 
Anhang

Tabelle 1: Wohnungsmerkmale und Quadratmeterpreis

\begin{tabular}{|l|l|l|}
\hline & Befragungsdaten & Wohnungsdatenbank \\
\hline Quartier & $0,14^{*}$ & $0,48^{* *}$ \\
& 2,13 & 7,96 \\
\hline Balkon & $0,15^{* *}$ & $0,22^{* *}$ \\
& 2,69 & 3,81 \\
\hline Holzfußboden & $0,19^{* *}$ & $0,35^{* *}$ \\
& 3,23 & 5,93 \\
\hline Wohnungsgröße in qm & $-0,07$ & $-0,28^{* *}$ \\
& $-1,10$ & $-5,23$ \\
\hline Loft & $0,22^{* *}$ & --- \\
\hline Stuckdecken & 3,89 & \\
\hline Einbauküche & $-0,02$ & --- \\
\hline Korrigiertes $\mathrm{R}^{2}$ & $-0,34$ & \\
\hline N & --- & $0,17 * *$ \\
\hline & & 3,07 \\
\hline & 0,21 & 0,61 \\
\hline
\end{tabular}

Lineare Regressionsmodelle. Angegeben sind die standardisierten Regressionskoeffizienten und die t-Werte. ${ }^{*} \mathrm{p}$ $<0,05 ; * * \mathrm{p}<0,01$ 
Tabelle 2: Mittelwerte und Standardabweichungen der verwendeten Variablen

\begin{tabular}{|c|c|c|c|}
\hline Variablenname & Erläuterung & Mittelwert & Standardabweichung \\
\hline Quartier & $\begin{array}{l}\text { Werte } 0 \text { und } 1, \quad \text { Quartier mit } \\
\text { Aufwertungstendenz }=1\end{array}$ & 0,45 & 0,498 \\
\hline Loftwohnung & Werte 0 und 1 & 0,097 & 0,297 \\
\hline $\begin{array}{l}\text { Wohnraum (in qm pro } \\
\text { Kopf) }\end{array}$ & & 38,94 & 15,507 \\
\hline Holzböden & Werte 0 und 1 & 0,64 & 0,482 \\
\hline Balkon & Werte 0 und 1 & 0,63 & 0,482 \\
\hline Stuckdecke & Werte 0 und 1 & 0,06 & 0,244 \\
\hline $\begin{array}{l}\text { Wohnung } \\
\text { preisgünstig }\end{array}$ & Werte 0 und 1 & 0,37 & 0,483 \\
\hline Schöne Wohnung & Werte 0 und 1 & 0,59 & 0,491 \\
\hline Schöne Gegend & Werte 0 und 1 & 0,41 & 0,492 \\
\hline Innenstadt & Werte 0 und 1 & 0,36 & 0,482 \\
\hline $\begin{array}{l}\text { Hochkultur- } \\
\text { orientierung }\end{array}$ & $\begin{array}{l}\text { Additive Skala aus Präferenz für } \\
\text { klassische Musik und Opernmusik }\end{array}$ & 2,97 & 1,108 \\
\hline Urbane Kultur & $\begin{array}{l}\text { Additive Skala aus der Häufigkeit } \\
\text { des Besuchs von Kinos, Kneipen, } \\
\text { Restaurants, Oper, Konzerthaus, } \\
\text { Kunstmuseum, Theater }\end{array}$ & 1,91 & 0,446 \\
\hline Geschlecht & Werte 0 (weiblich) und 1 (männlich) & 0,43 & 0,496 \\
\hline Alter & & 38,13 & 16,069 \\
\hline Partner & $\begin{array}{l}\text { Werte } 0 \text { und 1, Lebt die Person mit } \\
\text { einem Partner zusammen? }\end{array}$ & 0,47 & 0,499 \\
\hline Kinder & $\begin{array}{l}\text { Werte } 0 \text { und } 1 \text {, Lebt die Person mit } \\
\text { eigenen Kindern und/oder Kindern } \\
\text { des Partners zusammen? }\end{array}$ & 0,21 & 0,408 \\
\hline Bildung & $\begin{array}{l}\text { Werte } 1-4 \\
\begin{array}{l}\text { 1: Bis Hauptschulabschluss } \\
\text { 2: Mittlere Reife } \quad 26,4 \% \\
\text { 3: (Fach)hochschulreife } \quad 29,1 \% \\
\text { 4: (Fach)hochschulabschuss } 29,2 \%\end{array}\end{array}$ & 2,74 & 1,031 \\
\hline Einkommen in $1000 €$ & $\begin{array}{l}\text { Pro-Kopf Einkommen, gewichtet } \\
\text { nach neuer OECD Skala }\end{array}$ & 1,141 & 0,717 \\
\hline
\end{tabular}

